\title{
METAMORPHISM OF DRY SNOW AS A RESULT OF TEMPERATURE GRADIENT AND VAPOR DENSITY
}

\section{DIFFERENCES}

\author{
by \\ E. E. Adams and R. L. Brown \\ (Department of Civil Engineering and Engineering Mechanics, Montana State University, Bozeman,
} Montana 59717, U.S.A.)

\section{ABSTRACT}

A heat conduction equation for the determination of the temperature profile in a snowpack is developed. The magnitude of the temperature gradient tends to increase as the snow surface is approached, with local minima through layers of high snow density and local maxima above and below these layers. Calculations are made of the difference in vapor density in the pore and over the ice grain surfaces which border the pore. In the presence of sufficient temperature and temperature gradient, faceted crystals will develop near the top of the pore, as ice is sublimed away from the surfaces in the lower region. There will be a reduction in the percentage of rounded grains as the faceted form develops. The process is demonstrated to be enhanced at warm temperatures and large temperature gradients in low density snow.

\section{INTRODUCTION}

Temperatures at the base of a seasonal snowpack usually remain just below $0^{\circ} \mathrm{C}$ throughout the winter. Snow is warmed from below, as the ground gives up heat accumulated during the warmer months. If this geothermal heating coincides with cooler ambient air temperatures, characteristic of the winter months, a temperature gradient is established across the snowpack.

A variation in vapor pressure, resulting from the temperature differential, will cause a mass flux of vapor from the zone of high vapor pressure (the deeper warmer region) to the zone of lower vapor pressure. Since dry snow, in which this mechanism is most effective, consists of a solid and a vapor phase of the same material, the transfer can act as though it partly takes place through the solid matrix itself, as a hand-to-hand transfer of mass (Yosida and Kojima (1950), described in Akitaya 1974). Vapor will evaporate from the top of an ice grain, diffuse across the pore and deposit as a solid on the bottom of the grain above. The ice molecules on the top of this grain will sublime and redeposit in a similar fashion. When snow of low to medium density is subjected to the correct combination of temperature and temperature gradient, angular flat-surfaced crystals, known as depth hoar, will develop at the expense of those grains with a more rounded configuration. This effect is most prevalent in low-density snow at relatively warm temperatures subjected to large temperature gradients. Snowpack development in which faceted crystal growth predominates is known as temperature gradient metamorphism.

At low temperature gradients, rounded grains are observed to form, in a process known as equitemperature metamorphism. Colbeck (1982) refers to the crystals which develop as a result of equitemperature metamorphism as the equilibrium growth form. In the proper conditions this equilibrium form may be other than spherical. Colbeck terms the faceted crystals which develop as a result of temperature gradient metamorpnism the kinetic growth form. A satisfactory value for the temperature gradient at which the transition from the equilibrium to the kinetic growth form takes place has not been established; this is because of the dependency on temperature and snow density. The point at which this transition occurs is an important topic which requires further investigation. This paper deals with processes involved during kinetic growth.

Two general types of depth hoar are recognized (Akitaya 1974): a solid type, which develops under smaller temperature gradients, and a skeleton type, which predominates under large temperature gradients. Skeleton-type depth hoar is composed of large faceted crystals, which are poorly bonded together and form a weak snow layer. The solid type consists of small faceted crystals which do not show the marked reduction in strength characterized by the skeleton type. Akitaya (1974), in what is perhaps the most complete experimental work carried out on the topic of temperature gradient metamorphism, has shown that pore size, initial ice-grain size and geometry affects depth-hoar development. A large pore will enhance the rate of growth and the size of the crystal which will develop. This is in agreement with Marbouty's (1980) observation that fine-grained snow with a density in excess of approximately $350 \mathrm{~kg} \mathrm{~m}^{-3}$ subjected to a large temperature gradient will develop depth hoar of a solid type. Lower density snow, which has a large pore size, tends toward developing the skeleton type in the presence of a sufficient temperature gradient.

Faceted crystals grow toward the direction of higher temperature in the snow. The direction of crystal growth was observed to be dependent on the thermal gradient and not on gravity (Akitaya 1974). 
Several types of crystals will develop simultaneously in a particular layer with one form being predominant. The predominant crystal type was observed to be a function of temperature and temperature gradient (Akitaya 1974, Marbouty 1980).

An analytic development will be presented to describe some of the thermodynamic processes involved in temperature gradient metamorphism.

\section{HEAT AND MASS TRANSFER IN SNOW}

When all mechanical effects are neglected, the general isotropic form of the Fourier heat-conduction equation may be written as

$$
\frac{\partial}{\partial x}\left(k \frac{\partial T}{\partial x}\right)+\frac{\partial}{\partial y}\left(k \frac{\partial T}{\partial y}\right)+\frac{\partial}{\partial z}\left(k \frac{\partial T}{\partial z}\right)+Q=\rho_{S} C \frac{\partial T}{\partial t}
$$

where $k$ is the thermal conductivity, $T$ is temperature, $Q$ is the internal heat generation per unit time per unit volume, $\rho_{s} \mathrm{C} \partial \mathrm{T} / \partial \mathrm{t}$ is the rate of internal energy change per unit volume, $t$ is time, $\rho_{s}$ is the snow density, and $C$ is the specific heat of ice (Boley and Weiner 1960)

In the presence of a temperature gradient, heat flow in a natural snowpack occurs in the direction normal to the slope of the ground. This is taken to be the $z$ coordinate direction. There will be no flow of heat in the $x$ and $y$ component directions, which are taken as tangent to the slope, since the snow cover may be considered as extending infinitely in this plane. Using the ground-snow interface as the origin for the $z$ axis, a negative temperature gradient $\partial T / \partial z$ is established in the snowpack since the ground is, in general, warmer than the upper regions of the snow.

Internal heating within the snowpack may be generated during metamorphism as mass is sublimed from, and deposited onto, the ice surfaces. The net quantity of heat produced or lost may be calculated from the energy released due to the latent heat of sublimation, as the mass of water undergoes this phase transformation. Internal heat generation due to sublimation-deposition is

$$
Q=-L \frac{\partial J}{\partial z} \text {, }
$$

where $L$ is the heat of sublimation and $J_{S}$ is the mass flux of the snow.

Once again neglecting mechanical effects, a change in the snow density is the result of water vapor being driven upward through the snow. The conservation of mass in the snow may then be expressed by

$$
\frac{\partial J_{s}}{\partial z}=-\dot{\rho}_{s}
$$

where $\dot{\rho}_{S}$ is the time rate of change of snow density resulting from vapor flow.

Four years' data were collected in the highaltitude continental climate of Colorado, USA (Armstrong 1980), on the relation between compressive strain rates, thermal metamorphism, and density, for dry snow. Armstrong concluded that large differences in density which exist between snowpack developed by equi-temperature metamorphism and by temperature gradient metamorphism are the result of differential strain-rates, rather than significant mass flux due to diffusion.

Although there is probably some change in snow density as the result of diffusion alone, it is difficult to measure and is now considered to be slight. When mechanical effects such as compaction and settlement, and the thermal effects of vapor flow due to convection are neglected, the rate of change of the snow-density term can be ignored.
The thermal conductivity of ice is much greater than that of water vapor at the same temperature. Consequently, the major portion of heat transfer by pure conduction will take place through the solid ice network, rather than the pore. This is the reason why thermal conductivity is strongly dependent on snow density. However, part of the heat is carried upward by diffusion of water vapor through the pore as well. Because of this, an effective thermal-conductivity coefficient instead of the true conductivity is used to determine the temperature profile of the snowpack.

Empirical expressions which are generally used to determine the coefficient of thermal conductivity are based solely on snow density. (For a partial list of examples, see Mellor (1964).) Since density is certainly not the only parameter which influences conductivity, the data accumulated by Voitkovsky and others (1975) has been used. They obtained what they considered to be values near the true conductivity as a function of density, by taking measurements at very low temperatures, where diffusion is not such an effective means of heat transfer. Statistical methods were used to arrive at a true coefficient of thermal conductivity.

Data were also collected by Voitkovsky and others (1975) on conduction as a function of temperature. The relationship of the conductivity as a function of temperature is approximately exponential. Combining these results the equation in m.k.s. units which is used in this paper to evaluate the effective thermal conductivity coefficient of snow is

$$
\begin{aligned}
k= & \left(1.093 \times 10^{-3}\right) \exp (0.028 T)[0.030+ \\
& +0.303\left(\rho_{S} \times 10^{-3}\right)-0.177\left(\rho_{S} \times 10^{-3}\right)^{2}+ \\
& \left.+2.250\left(\rho_{S} \times 10^{-3}\right)^{3}\right] .
\end{aligned}
$$

Although this ignores textural considerations the expression does use two important parameters which are easily measurable.

No data are available which indicate the dependence of thermal conduction on intergranular bonding. This is unfortunate since the manner in which the bonds, or necks, join the ice grains must be significant to thermal conduction in the snow. Well-bonded snow with large necks will facilitate heat flow more readily than poorly sintered snow of the same density, since the conductivity of ice is higher than that of water vapor or air.

Substituting $-\dot{p}_{s}$ (which is neglected) from Equation (3) for $\partial J_{s} / \partial z$ in Equation (2) and recognizing that the coefficient of thermal conductivity for snow will vary with position in the snowpack, a Fourier equation for heat conduction in snow is

$$
k \frac{\partial^{2} T}{\partial z^{2}}+\frac{\partial k}{\partial z} \frac{\partial T}{\partial z}=\rho_{S} C \frac{\partial T}{\partial t},
$$

VAPOR PRESSURE IN THE PORE

Since depth-hoar crystals are grown from the vapor phase, development of these crystals in a snowpack must be restricted to the pores. It is the surfaces of the ice grains which comprise the pore boundaries that provide the nucleation sites for crystal growth. Differences in vapor density in a pore with an imposed temperature gradient are examined in an effort to understand the relationship between depthhoar crystal development at the expense of the rounded ice grains and the preferential direction of faceted crystal growth. A schematic representation of the pore for this one-dimensional flow analysis is shown in Figure 1 .

The mass flux of water vapor across a pore in the presence of a temperature gradient can be described by

$$
J=-\frac{D}{R T} \frac{d P}{d z},
$$

where $J$ is the mass flux of water vapor across the 


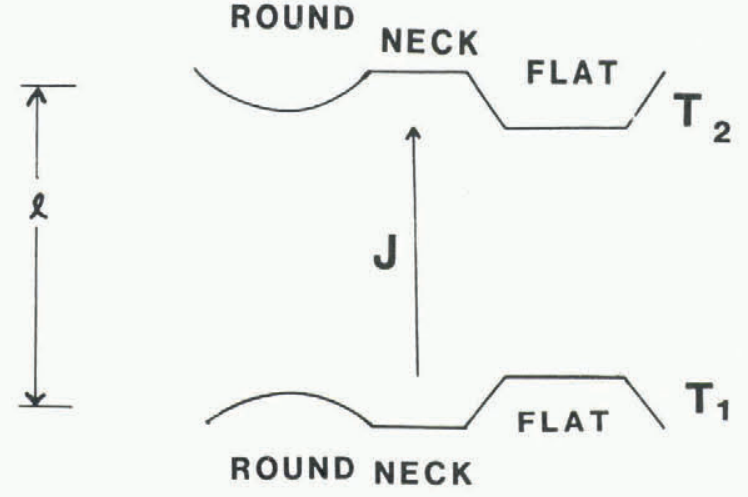

Fig.1. A schematic representation of the idealized pore which is used in the model. $\ell$ is the pore height and taken to be the same for all shapes, $T$ is temperature and $J$ is mass flux. The direction of $\mathrm{J}$ shown is valid for $T_{1}>T_{2}$.

pore, $D$ is the coefficient of diffusion, $R$ is the gas constant for water vapor, $T$ is temperature, $P_{v}$ is the vapor pressure in the pore, and $z$ is the coordinate direction in which the temperature gradient acts. From continuity we have

$$
\frac{d J}{d z}=-\dot{\rho}_{v} \text {, }
$$

where $\dot{\rho}_{v}$ is the time rate of change of vapor density in the pore. Vapor density is taken to be constant for steady-state conditions at any particular temperature and height in the pore; $\dot{p}_{y}=0$.

The diffusion coefficient is dependent on temperature (Giddings and LaChapelle 1962) as

$$
D=\alpha T^{1.7},
$$

where $\alpha$ is a proportionality constant.

Equations (6), (7) and (8) can be combined to give

$$
\left.\frac{d}{d z}{\frac{\alpha T^{\bullet}}{R}}^{7} \frac{d P_{v}}{d z}\right]=0
$$

Assuming a constant temperature gradient across the pore, water vapor pressure as a function of temperature and position in the pore is

$$
P_{v}=\frac{A R}{\alpha} \frac{3.333}{\frac{d T}{d z}}\left[T(z) \cdot 3-T_{0} \cdot 3\right]+P_{v_{0}},
$$

where $A$ is a constant determined by pore boundary conditions, $P_{\text {vo }}$ is the vapor pressure at the bottom of the pore, and $T_{0}$ is the temperature at the bottom of the pore.

Each pore boundary is composed of a variety of crystal-surface shapes. Two basic shapes are examined here: grains with a predominately rounded configuration and faceted crystals composed of flat surfaces.

Colbeck (1980) states the equilibrium vapor pressure $P$ over the surface of an ice grain varies with temperature and mean radius of curvature. A relationship between saturation vapor pressure and temperature may be found by using the ClausiusClapeyron equation for a particular radius of curvature. The vapor pressure in terms of the mean radius of curvature at a fixed temperature may be examined by Kelvin's equation. A single expression for the saturation vapor pressure over the grain surfaces can be found by combining these equations. The saturation vapor pressure in terms of the temperature $T$ and mean radius of curvature $r$ becomes

$$
\left.P(T, r)=P^{-} \exp \left[\frac{2 \sigma}{\rho_{j} R T^{-} r}\right] \exp \left[\frac{L}{R} \frac{(1}{T^{-}}-\frac{1}{T}\right)\right],
$$

where $P^{-}$and $T^{-}$are the reference pressure and temperature, $\rho_{i}$ is the density of ice, and $\sigma$ is the interfacial energy.

Vapor pressure over the ice surfaces which comprise the upper and lower boundaries of the pore are calculated from Equation (11). These results give the boundary conditions which are used to solve for the constants $A$ and $P_{\text {vo }}$ in Equation (10), for each radius of curvature. The vapor pressure at the upper and lower boundaries of the pore is determined by the temperature and the relative densities of grains with rounded and faceted geometry and of the necks. These all contribute to the vapor pressure at the pore boundaries. The total vapor pressure in the pore is the sum of the contributions of ice grains with a particular radius of curvature and of the necks. The mean vapor pressure in the pore can now be calculated by evaluating Equation (10) at the center of the pore for each radius of curvature, and the density of crystals with that particular radius of curvature. In this way, the effect of the different grain radii are weighted by their relative densities. Explicitly, the vapor pressure at the center of the pore is

$P_{v}=\sum_{m=1}^{n} \frac{A_{m} R}{\alpha}\left[\begin{array}{l}\frac{3.333}{(d T)} \\ d z\end{array}\left[\frac{(l)^{.3}}{2}-T_{0}^{.3}\right]+P_{v o}(m)\right] \frac{m}{\rho S}$

where the subscript $m$ indicates a particular radius of curvature, $n$ is the total number of different radii, $\rho_{m}$ is the density of crystals with $r_{m}$ radius, $\rho_{S}$ is the density of snow, and $\ell$ is the neight of the pore.

It should be noted here that the solution obtained from Equation (10) gives a nonlinear variation in the vapor pressure as $z$ is varied from 0 at the bottom of the pore to $l$ at the top of the pore. This effect shows up in the final calculation of vapor pressure by Equation (12). Physically, the temperature gradient induces a slight bulge in the pore vapor pressure near the center of the pore.

Vapor-density difference is defined here to be the difference between the vapor density in the center of the pore and that over the ice surface calculated at equilibrium. The vapor-density difference term is arrived at by dividing the pressure difference from Equations (12) and (11) by the gas constant for water vapor and the temperature at the ice surface.

The maximum vapor-density difference within the pore will determine the predominant type of crystal which will form, because growth will proceed most readily here. This maximum vapor-density difference will be at the top of the pore over the flat ice surfaces. Crystal development which occurs at low supersaturations will have the form of columns or plates, depending on temperature. Therefore, at sufficient supersaturations for the kinetic growth forms, the predominant new growth in the pore will be in the form of faceted crystals, at the top of the pore. Profiles of maximum vapor-density differences which are shown graphically are calculated in this region. If only rounded surfaces and necks are present initially, in the presence of sufficient supersaturation, faceted crystal growth will develop on the rounded surfaces and flat surfaces will be produced.

\section{RESULTS AND CONCLUSIONS}

In order to examine the vapor densities associated with various crystal shapes, a simplified pore geometry is assumed. The rounded grains are modeled as spheres. The flat faced crystals are assumed to have an infinite radius of curvature. Although calculating the vapor densities over the flat surfaces 


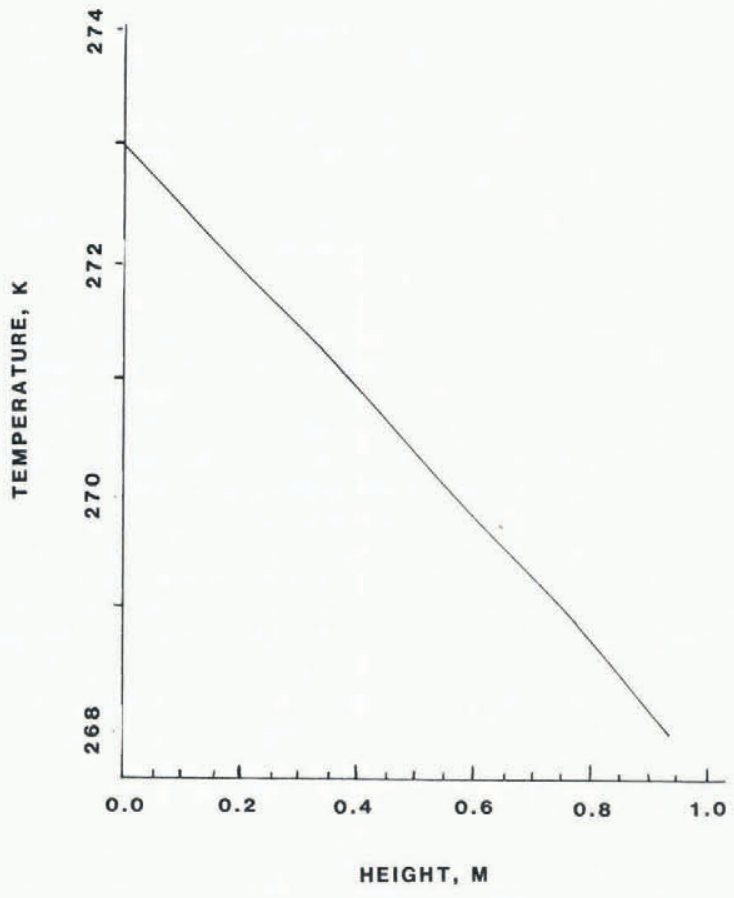

Fig.2. Calculated temperature profile for snow of homogeneous density of $100 \mathrm{~kg} \mathrm{~m}^{-3}$ which has a temperature difference of $5 \mathrm{deg}$ between the top and bottom boundaries.

is an approximation, the supersaturations necessary to sustain the kinetic growth form dominates the effect of crystal geometry for crystals greater than $1 \mu$ (Frank 1958:310, Colbeck 1982).

Mean pore size is determined by snow density and the size of the ice grains. A linear regression routine was employed to calculate the pore size, and was based on a limited number of thin sections taken by Akitaya (1974). Results presented in this section assume snow composed predominantly of spherical grains with a mean radius of curvature of $0.5 \mathrm{~mm}$. With this radius, a pore height of $1.6 \mathrm{~mm}$ results for snow with a density of $400 \mathrm{~kg} \mathrm{~m}^{-3}$ and $3.9 \mathrm{~mm}$ for $100 \mathrm{~kg} \mathrm{~m}^{-3}$ density snow.

Results from Equation (5) are first examined by considering homogeneous snow. In doing so, the effect which temperature has on the thermal conductivity and, hence, on the temperature profile, may be examined. Snow with a density of $100 \mathrm{~kg} \mathrm{~m}^{-3}$ is subjected to several different surface temperatures. With a differential of $5 \mathrm{deg}$, a nearly linear temperature distribution with depth is produced (Fig.2). As the difference in temperature is increased, the deviation from a linear temperature profile increases (Fig.3).

Figure 4 is the temperature-gradient distribution corresponding to the temperature profile in Figure 3 . Results displayed in Figure 4 indicate that the magnitude of the temperature gradient becomes larger as the snow surface is approached from the bottom. This is in agreement with Akitaya's (1974) observations in a natural snowpack.

An analysis of Figure 5, which is for the homogeneous snow with large differential boundary temperatures (Fig.3), shows that the maximum vapordensity difference decreases at lower temperatures, even in the presence of an increasing magnitude of temperature gradient. Compare Figures 3,4 and 5 . This demonstrates analytically why depth hoar is observed to grow more readily in the lower regions of the snowpack than it does in the upper regions, even when the negative temperature gradient is greater in magnitude at the higher elevation.

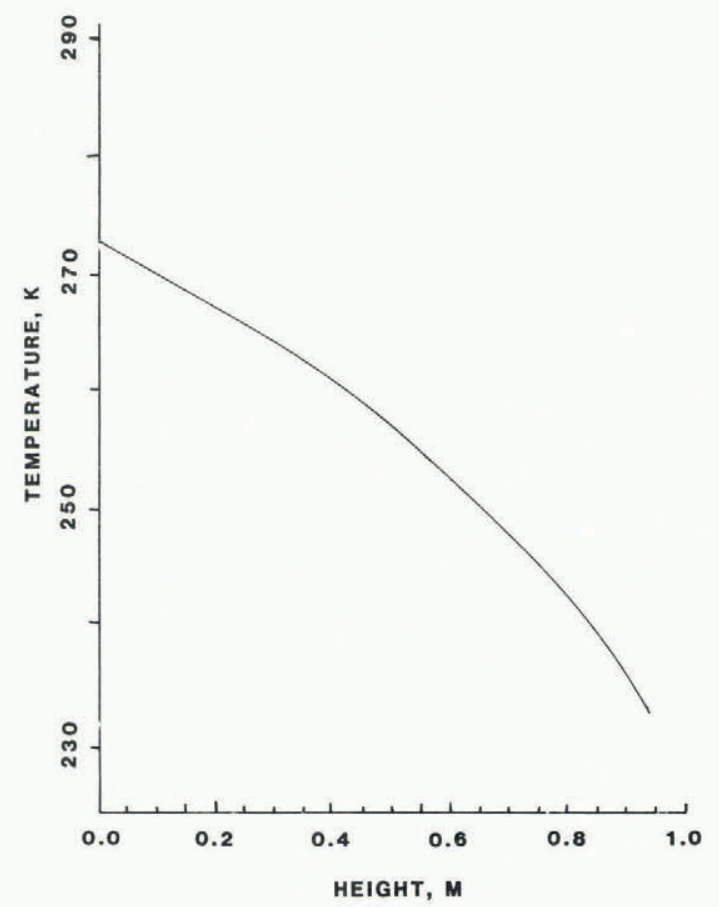

Fig.3. Calculated temperature profile for snow of homogeneous density of $100 \mathrm{~kg} \mathrm{~m}^{-3}$ which has a temperature difference of 40 deg between the top and bottom boundaries (example 1).

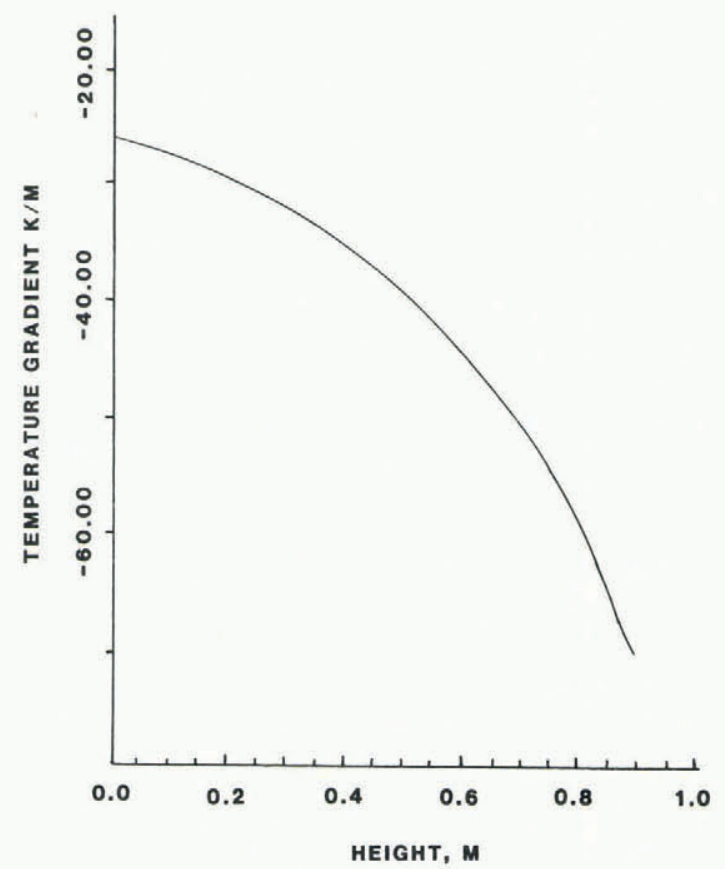

Fig.4. Temperature gradient profile corresponding to the temperature profile in Figure 3 (example 1 ).

Further insight into the temperature-gradient metamorphic process is obtained when vapor-density differences over the rounded surfaces, and the flat surfaces at the top and bottom of the pores are examined. There is an overall positive vapor-density difference over the ice surfaces in the upper region of the pore and a negative difference over the surfaces on the bottom. This causes a migration of mass from the lower to the upper regions of the pore. 


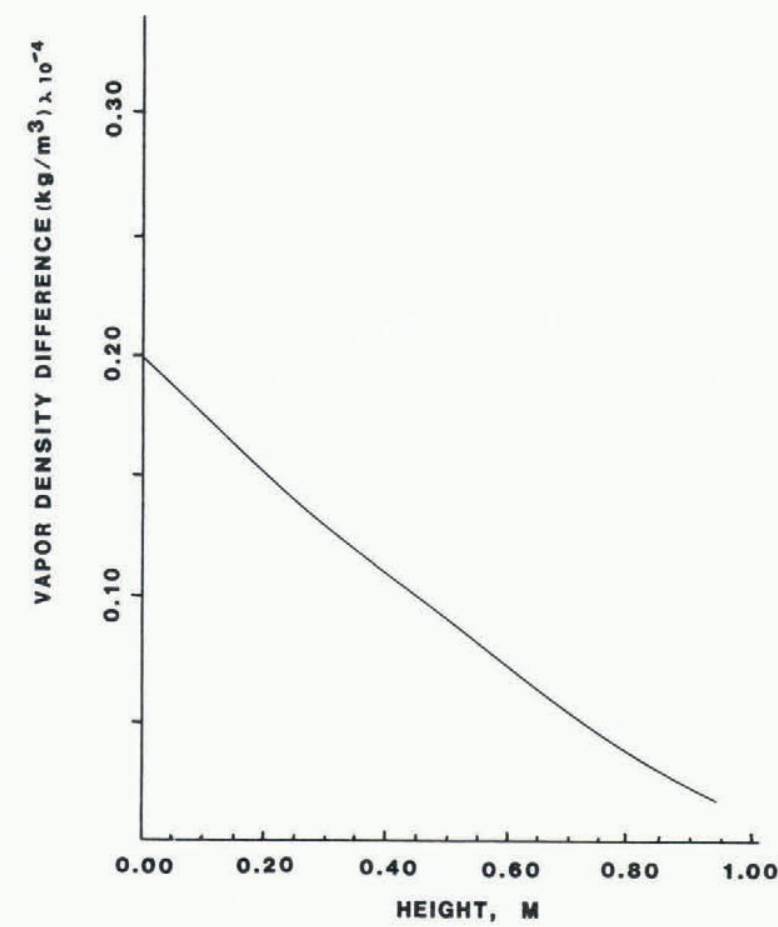

Fig.5. Maximum vapor-density difference profile (which is taken over the flat surfaced crystals at the top of the pore) in the homogeneous snowpack (example 1).

This process is in agreement with the hand-to-hand transfer of mass studied by Yosida and Kojima and described in Akitaya (1974). The imbalance in vapor density also accounts for the preferential direction of growth of depth hoar crystals as observed by Akitaya (1974).

The negative vapor-density difference over the ice surfaces in the lower region of the pore is greatest over the rounded (depending on radius) and least over the flat surfaces. A negative vapordensity difference causes the sublimation of mass away from the ice surfaces involved. Rounded ice grains near the bottom of the pore will sublime more readily than the flat surfaces at the same level. At sufficient supersaturation new development in the pore will form either plates or columns. As a result, there is a decline in the density of rounded grains, while the density of the faceted form increases.

Homogeneous snowpacks rarely exist in nature. Layers of dense snow resulting from wind, sun, rain, atmospheric conditions of deposition, etc., are usually present to form a more complex stratigraphy. Effects of this density layering on metamorphism is examined next. Sharp contrasts in density are used so that pronounced effects of layering may be examined.

A dense layer placed near the bottom of the snowpack will cause a rise in the maximum vapor-density difference (compared to the homogeneous snow) on both sides of the crust. In this case the layer produces a major increase in the vapor density difference above the crust. Faceted crystal growth associated with the zones of the high vapor-density differences shows excellent correlation with observations in a natural environment. Development of depth hoar crystals in the field are known to occur both immediately above and below crusts. Frowth above the layers is common and is most frequently seen when the temperature is near $273 \mathrm{~K}$ (Perla and Martinelli 1976, Adams and Brown 1982).

The effect of several layers of high-density snow in a close proximity to each other is shown in Figures 6 to 9 . Higher thermal conductivity associated

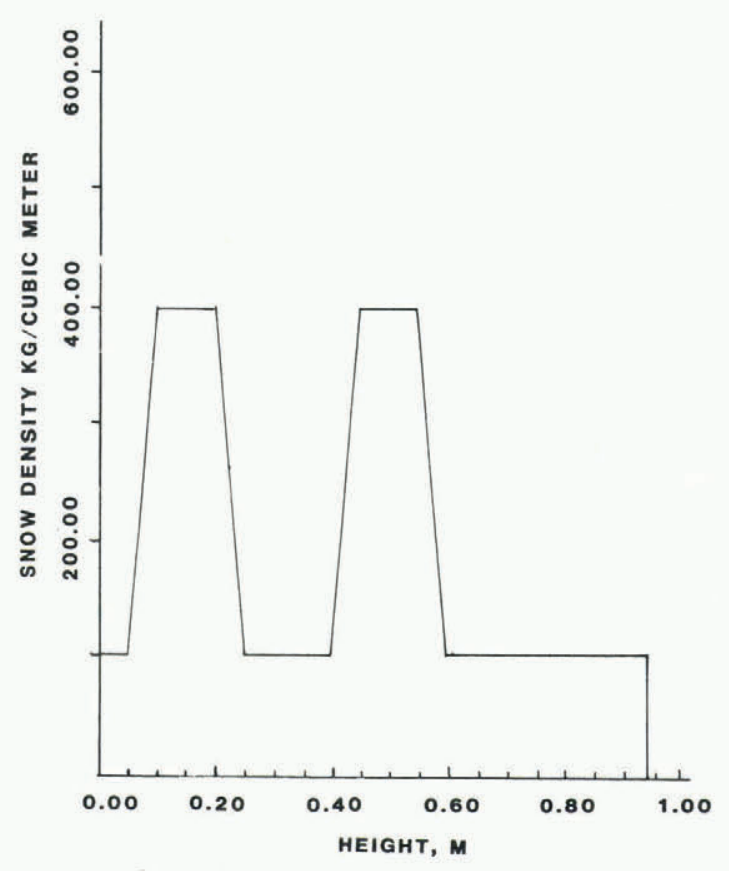

Fig.6. Density profile of a theoretical snowpack with a layered stratigraphy (example 2).

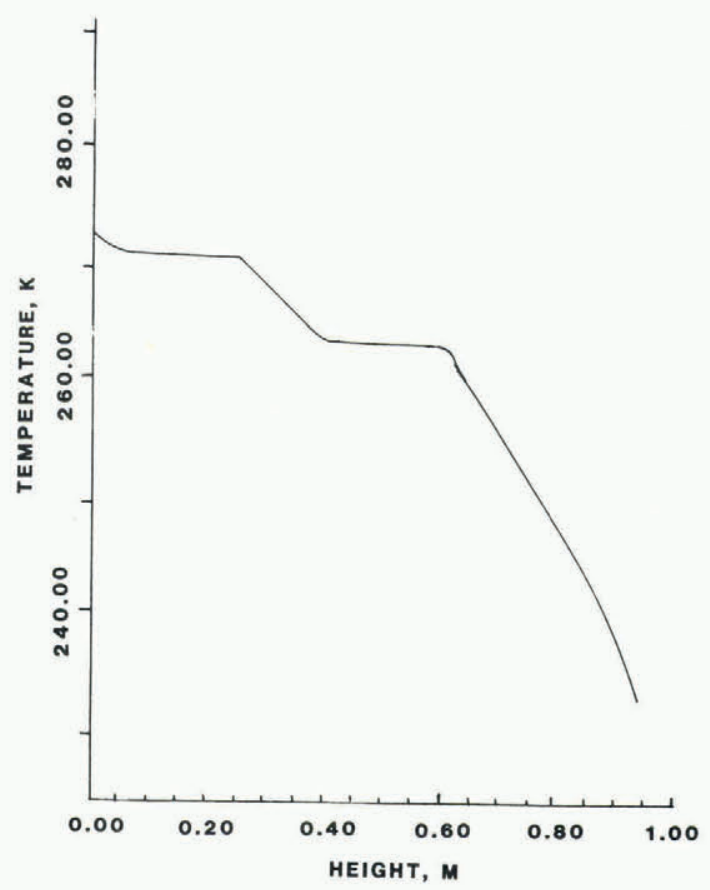

Fig.7. Calculated temperature profile for the densitylayered snowpack represented in Figure 6 (example 2).

with the more dense snow layers causes warmer temperatures to be maintained from the base through the upper dense layer in Figure 7. As demonstrated previously, higher vapor-density differences are sustained at warmer temperatures.

A combination of the increase of the vapordensity difference above the lower dense snow layer and below the upper dense snow layer causes a large vapor-density difference to be produced in the low density snow between these layers (Fig.9). This effect is heavily increased by the large negative temperature gradient (Fig.8) and relatively warm 


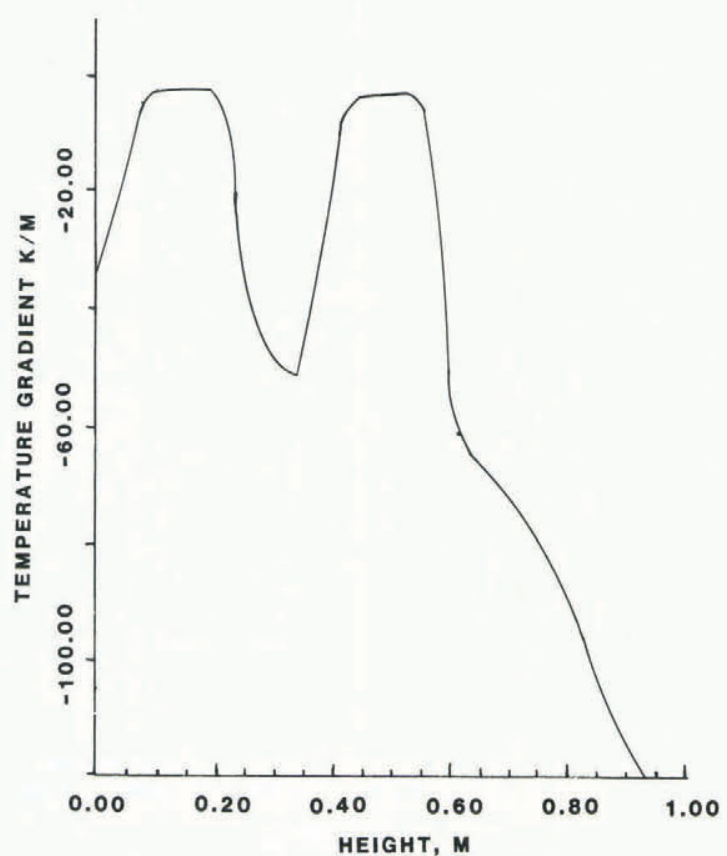

Fig.8. Temperature gradient profile corresponding to the temperature profile in Figure 7 (example 2 ).

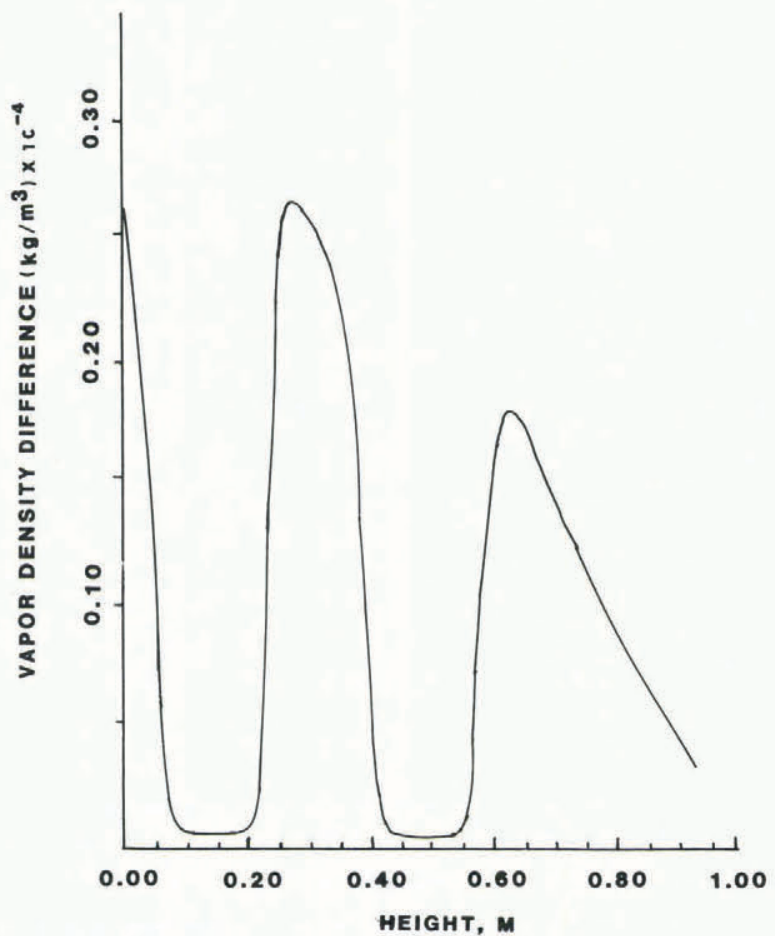

Fig.9. Maximum vapor-density difference (which is taken over the flat surfaced crystals at the top of the pore) in the snowpack with a layered stratigraphy (example 2).

temperatures (Fig.7) involved in this zone. It is also the large negative temperature gradient and warm temperatures which causes the pronounced spike in the vapor density difference profile just above the upper high density snow layer. Since the vapordensity difference facilitates the growth of depth hoar, it may be assumed that when a low density snow is sandwiched between two higher density layers, at sufficiently warm temperatures, faceted crystal growth will be enhanced between the layers. The vapor- density differences through the dense layers are very low, indicating that faceted crystal growth is less prevalent here. This is true, because of the smaller pore size associated with higher density snow, as well as the smaller temperature gradient produced by the higher thermal conductivity.

It may be noted at this point, that an increase in magnitude of the temperature gradient will cause a greater vapor-density difference in the upper region of the pore and a more negative vapor-density difference in the lower region, if all other parameters, such as temperature, density, and grain size remain the same. This situation may be examined in part by referring to depth 0.00 in Figures 5 and 9 . Development of faceted crystals, and a decrease in the density of rounded grains is enhanced when the magnitude of the temperature gradient is increased.

Initial grain size and pore size of a snow sample also have an effect. Snow with grains which have a smaller radius of curvature will also have a smaller pore space than a snowpack with larger grains but the same density. The vapor-density difference curves for two samples which are of the same snow density, but of different grain size are essentially the same, but the values are lower for the smaller grained snow. Fine-grained snow also has a smaller magnitude of negative vapor-density difference at the bottom of the pore. These results indicate that fine-grained snow will be less susceptible to depth hoar development than coarse-grained snow of equal snow density, because of the correlation of the grain size to the pore size.

A more accurate description of the pore size based on geometric constraints of the snow, such as grain size, crystal type, and snow density is needed. This would best be accomplished by a very detailed experimental examination of the pore space for a wide variety of snow types. Other areas which warrant further experimental investigations are the assumptions of a linear temperature profile across the pore and of constant snow density with time.

The theory presented in this paper works well in describing some of the processes which accompany what is generally known as temperature gradient metamorphism. However, more work needs to be carried out before all of the principles which govern it can be adequately described. The model presented indicates that a reasonable approach to the solution may involve a very detailed and accurate analysis of the processes involved within a single pore.

\section{ACKNOWLEDGMENT}

The work reported in this paper was funded by NSF Grant No.ENG-7901636. The authors wish to express their appreciation to the US National Science Foundation for their support.

\section{REFERENCES}

Adams E E, Brown R L 1982 Further results on studies of temperature-gradient metamorphism. Joumal of Glaciology 28(98): 205-210

Akitaya E 1974 Studies on depth hoar. Contributions from the Institute of Low Temperature Science Ser A 26

Armstrong R L 1980 An analysis of compressive strain in adjacent temperature-gradient and equitemperature layers in a natural snow cover. Journat of Glaciology 26(94): 238-289

Boley B A, Weiner J H 1960 Theory of thermal stresses. New York, John Wiley and Sons

Colbeck S C 1980 Thermodynamics of snow metamorphism due to variations in curvature. Jourmal of Glaciology 26(94): 291-301

Colbeck S C 1982 An overview of seasonal snow metamorphism. Reviews of Geophysics and Space Physics 20(1): $45-61$

Frank F C 1958 Introductory lecture. In Doremus $\mathrm{R} \mathrm{H}$, Roberts B W, Turnbul1 D (eds) Growth and perfection of crystals. New York, John Wiley 
Giddings J C, LaChapelle E 1962 The formation rate of depth hoar. Journal of Geophysical Research $67(6): 2377-2383$

Marbouty D 1980 An experimental study of temperature-gradient metamorphism. Journal of Glaciology 26(94): 303-312

Mellor M 1964 Properties of snow. CRREL Monograph II I-A1

Perla R I, Martinelli M Jr 1976 Avalanche handbook. US Department of Agriculture. Forest Service. Agriculture Handbook 489

Voitkovsky K F, Golubev V N, Lapteva N I, Troshkina E S, Ushakova L A, Pavlov A V 1975 Mass transfer and metamorphism in snow cover. Intermational Association of Hydrological Sciences Publication 114 (Symposium of Grindelwald 1974 Snow Mechanics): $16-24$ 